\title{
Red-Hed regulation: recombinase Rad51, though capable of playing the leading role, may be relegated to supporting Dmc1 in budding yeast meiosis
}

\author{
Sean Sheridan ${ }^{1}$ and Douglas K. Bishop ${ }^{1,2,3}$ \\ ${ }^{1}$ Committee on Genetics, Department of Molecular Genetics and Cell Biology, University of Chicago, Chicago, Illinois, \\ USA; ${ }^{2}$ Department of Radiation and Cellular Oncology, Department of Molecular Genetics and Cell Biology, University of \\ Chicago, Chicago, Illinois, 60637 USA
}

DNA strand exchange is the central process of homologous recombination. In budding yeast, this reaction is catalyzed by the recombinases Rad51 and Dmc1. Rad51 is responsible for recombinational repair of DNA damage during mitosis and is also important during meiotic recombination. Dmc1 is only expressed during meiosis and is required for meiotic recombination. The discovery that these two different recombinases are needed for normal levels of meiotic recombination in budding yeast raised the question of how the functions of these proteins relate to one another. The paper by Tsubouchi and Roeder (2006) in this issue of Genes \& Development represents important progress toward what is apparently a rather complex answer.

Tsubouchi and Roeder (2006) report discovery of a novel meiosis-specific protein, Hed1, which appears to inhibit Rad51 when Dmc1 is absent. The authors show mutation of the HED1 gene allows cells to bypass the meiotic arrest caused by a $d m c 1$ mutation and resolve meiosis-specific double-strand breaks (DSBs) using Rad51. A hed1 mutation can also suppress the defects caused by mutation of HOP2, which codes for a Dmc1 accessory factor. Two-hybrid experiments show that Hed 1 and Rad51 can interact directly and immuno-staining shows that subnuclear foci formed by Hed1 and Rad51 colocalize. Together these results imply that Hed1's influence on Rad51 activity is direct. Furthermore, expression of Hed1 in mitotic cells provided evidence that Hed1 expression can be sufficient to inhibit Rad51-dependent repair of mitotic DNA damage.

A reasonable interpretation of these results is that the meiotic recombination machinery of budding yeast is regulated such that Dmcl carries out most strand ex-

${ }^{3}$ Corresponding author.

E-MAIL dbishop@uchicago.edu; FAX (773) 834-9064.

Article is online at http://www.genesdev.org/cgi/doi/10.1101/gad.1447606. change. Tsubouchi and Roeder's (2006) results also add to evidence indicating that Rad51 is capable of replacing Dmc1's function under certain circumstances. Here, we place these new findings in the context of previous observations and present a model to explain how Rad51 and Dmc1 may cooperate to promote interhomolog recombination in budding yeast, while at the same time accounting for the observed functional redundancy. We further speculate as to how the functional relationship of Rad51 and Dmc1 may have evolved.

The notion that Rad51 activity is blocked during meiosis is not new. Previous work showed that Dmc1independent recombination is inhibited by proteins associated with axial elements (Schwacha and Kleckner 1997; Xu et al. 1997; Bishop et al. 1999; Niu et al. 2005) proteinaceous structures that organize pairs of sister chromatids into two parallel sets of loops by binding at their base. The assembly of the synaptonemal complex brings an axial element with its pair of sisters into close parallel alignment with another axial element holding the homologous sister pair. Three interacting axis-associated proteins-Red1, Hop1, and Mek1-regulate recombination by suppressing Dmcl-independent recombination and, in the case of Red1 and Hop1, by enhancing the efficiency of DSB formation in a locus-specific manner (Rockmill and Roeder 1990; Mao-Draayer et al. 1996; Schwacha and Kleckner 1997; Pecina et al. 2002). Red, Hop1, and Mek1 also influence the choice of recombination partner (as discussed in more detail below). In comparison to the axis-associated proteins, the properties reported for Hed1 indicate that it has a more direct and specific role in Rad51 inhibition (Tsubouchi and Roeder 2006).

\section{Similarities between the oligomeric forms} and biochemical activities of Rad51 and Dmc1

Rad51 and Dmc1 have similar recombinase activity. The initial evidence that Rad51 was a structural and func- 
tional relative of RecA came from mutant analysis, detection of Rad51's DNA-dependent ATPase activity, and the observation that the protein forms right-handed helical filaments on DNA (Shinohara et al. 1992; Ogawa et al. 1993; Benson et al. 1994). Dmcl was also proposed to be a structural and functional relative of RecA based on mutant and sequence analysis (Bishop et al. 1992; Story et al. 1993). Biochemical demonstration of recombinase activity for Rad51 and Dmcl supports the idea that each protein promotes strand exchange by a mechanism related to that of RecA (Sung 1994; Baumann et al. 1996; Li et al. 1997; Masson et al. 1999; Hong et al. 2001). Although similar in several respects, electron microscopy (EM) analysis showed that Dmc1 and Rad51 differ in that Dmcl has a strong tendency to form tori and can bind DNA in the toroid form (Masson et al. 1999; Passy et al. 1999; Kinebuchi et al. 2004). This led to the suggestion that Dmc1 might promote recombination via a mechanism distinct from that of Rad51 and RecA. However, subsequent studies showed that, under certain conditions, Dmc1 forms right-handed filaments similar to those formed by the other recombinases (Sehorn et al. 2004; Burgreev et al. 2005; Lee et al. 2005). Interestingly, substitution of $\mathrm{Mg}^{2+}$ with $\mathrm{Ca}^{2+}$ resulted in formation of long helical filaments on single-stranded DNA (ssDNA); $\mathrm{Ca}^{2+}$ stimulated Dmcl's strand assimilation and strand exchange activity as well (Bugreev et al. 2005; Lee et al. 2005). Although it remains to be seen if $\mathrm{Ca}^{2+}$ is a biologically relevant modifier of Dmcl activity, it is clear that conditions favoring filament formation enhance recombinase activity. Thus, as with RecA and Rad51, the functional form of Dmcl is almost certainly the helical filament. Overall, the intrinsic biochemical properties of Rad51 and Dmc1 determined to date appear quite similar, consistent with in vivo observations indicating the ability of these proteins to substitute for one another.

Although the intrinsic activities of purified recombinases are similar, the in vivo function of the two proteins is controlled by distinct sets of accessory factors. Assembly of Rad51 at DSB sites is promoted by both Rad52 and Rad55-Rad57 (Gasior et al. 2001), while assembly of Dmc1 is partially dependent on Rad51 (Bishop 1994; Shinohara et al. 1997) and its activity also depends on the Sae3-Mei5 protein (Hayase et al. 2004; Tsubouchi and Roeder 2004). Two additional factors, Rad54 and Tid1/ Rdh54, are capable of stimulating the activity of both recombinases (Tan et al. 2003; E. Hong and D. Bishop, unpubl.), although Rad54 may normally partner with Rad51 and Tid1/Rdh54 with Dmc1. Finally, in vivo experiments suggest another accessory protein, Hop2Mnd1, stimulates Dmc1-dependent and not Rad51-dependent recombination in vivo (Tsubouchi and Roeder 2003; Chen et al. 2004; Henry et al. 2006). Although these accessory factor differences may allow for distinct regulation of the two recombinases, we reiterate that the ability of each of the recombinases to promote substantial strand invasion in the absence of the other indicates a significant degree of functional redundancy in vivo (Schwacha and Kleckner 1997; Shinohara et al. 1997;
Zenvirth et al. 1997; Bishop et al. 1999; Tsubouchi and Roeder 2003).

\section{Rad51 and Dmc1 often function together}

In spite of the fact that Rad51 and Dmc1 can substitute for one another in mutant backgrounds, a number of observations suggest that both recombinases act together. Immunostaining of chromosome spreads showed that both Rad51 and Dmc1 form DSB-dependent immunostaining foci, most of which colocalize, at the time when meiotic strand exchange occurs (Bishop 1994). ImmunoEM studies confirmed this colocalization pattern in mouse spermatocytes (Tarsounas et al. 1999). In budding yeast, the normal appearance of Dmcl foci depends on Rad51 (Bishop 1994; Shinohara et al. 1997) and disappearance of Rad51 foci depends on Dmc1. These results are consistent with a single pathway in which the functions of the two recombinases are interdependent. Both recombinases are needed for the interhomolog connections between paired axial elements that are seen in SCdefective zip1 mutants, again consistent with cooperation of the recombinases (Rockmill et al. 1995). Finally, analysis of Rad51- and Dmc1-dependent recombination intermediates provided evidence that unique functions of Rad51 and Dmc1 contribute to normal progression of a recombination event (Schwacha and Kleckner 1997). Differences in the relative strengths of rad51 and dmc1 single mutant phenotypes further support the conclusion that the two recombinases have distinct functions (Shinohara et al. 1997). All of these results lead to the view that a major recombination pathway involves both recombinases. However, the possibility that a substantial subset of normal recombination events proceed via a pathway(s) involving only one of the two recombinases has not been excluded.

\section{Interhomolog vs. intersister recombination}

The most fundamental difference between meiotic and mitotic recombination is the type of partners involved in the exchange reaction. The vast majority of mitotic recombination events occur between a pair of sister chromatids (Kadyk and Hartwell 1992), while meiotic recombination favors exchange between homologous chromatids (Jackson and Fink 1985). Interhomolog recombination is fundamental to meiosis as it creates the physical connection between homologs necessary for proper chromosome segregation. Given that the change from sister to homolog recombination preference is a key feature of the meiotic program, it has been attractive to suppose that Dmc1, the meiosis-specific recombinase, evolved to serve this function. This may be true, but the current situation in budding yeast appears more complicated. On the one hand, it is clear that interhomolog recombination is strongly impaired in the $d m c 1$ mutant. On the other hand, the need for Dmc1 in interhomolog recombination can be efficiently bypassed by enhancing the activity of Dmc1-independent recombination either 
by elevating expression of Rad54, an accessory factor that stimulates Rad51 activity (Bishop et al. 1999), by elevating expression of Rad51 itself (Tsubouchi and Roeder 2003), or, as Tsubouchi and Roeder (2006) have now shown, by removing Hed1. Thus both recombinases can promote interhomolog partner choice. What follows is a discussion of evidence indicating that cooperation of the two recombinases is responsible for the high level of interhomolog partner choice observed in wild-type yeast.

Both recombinases contribute to interhomolog activity during normal meiosis

Development of two-dimensional (2-D) gel methods made it possible to detect the homologous joint molecule intermediates formed by recombinases at a particularly strong meiotic recombination hotspot, and allowed joints formed between homologous chromosomes to be distinguished from those that form between sister chromatids (Schwacha and Kleckner 1994). Although originally thought to represent intermediates leading to formation of both crossover and noncrossover recombinants, subsequent studies indicate that the joint molecules detected by 2-D gel are primarily precursors to the formation of reciprocal crossover recombinants (Allers and Lichten 2001; Hunter and Kleckner 2001; Borner et al. 2004). Nonetheless, joint molecule analysis revealed several features of the role of recombinases in partner choice selection.

Determination of the role of Dmcl in promoting interhomolog over intersister recombination was complicated by the fact that the $d m c 1$ mutant formed almost no detectable joint molecules, sister or homolog. However, mutation of RED1 allows formation of Dmc1-independent joints and thereby allows examination of the role of each of the recombinases in promoting the residual interhomolog recombination observed in this mutant. This approach demonstrated that Dmc1 has a function that promotes interhomolog partner choice that Rad51 lacks (Schwacha and Kleckner 1997).

Although Dmc1 has an interhomolog recombination activity that Rad51 lacks, there is also evidence (sometimes overlooked) that Rad51 is required for the strong homolog bias seen in wild-type cells. In a rad51 single mutant, the efficiency of DSB repair is reduced (Shinohara et al. 1992), but the intermediates that are formed show an eightfold decrease in the ratio of interhomolog to intersister joint molecules (Schwacha and Kleckner 1997). These data emphasize the importance of Rad51 in promoting interhomolog partner choice. It should also be reiterated that the Dmc1-specific interhomolog activity is not essential for budding yeast to achieve high levels of interhomolog recombination. This conclusion is based on the high level of recombination seen with overexpression of Rad54 or Rad51 in dmc1 mutants (Bishop et al. 1999; Shinohara et al. 2003; Tsubouchi and Roeder 2003) and is further supported by the high degree of interhomolog recombination observed by Tsubouchi and Roeder (2006) in the hed1 dmc1 double mutant.
Another important observation is that even when both recombinases are intact, mutation of the axial proteins can reduce the efficiency of interhomolog partner choice (Schwacha and Kleckner 1997; Wan et al. 2004; Niu et al. 2005). This indicates that cooperation of recombinases is not sufficient for normal partner choice. Therefore Dmc1 and Rad51 act in concert with axial proteins to promote interhomolog over intersister recombination.

\section{Relationship between axial proteins and Hed1}

Red1 influences recombination at the DSB stage as well as during strand invasion. The pleiotropic effects of corresponding mutants complicated interpretation of the role of Red1 in regulating Dmc1-independent recombination. Recent work with an inhibitor-sensitive allele of the MEK1 gene, mek1-as, clarified the role of axial proteins in recombination suppression. MEK1 is not required for normal levels of DSB formation (Xu et al. 1997). In addition, other work showed that a mek1-null mutant does not cause the drastic effect on axial structure seen in a red1-null mutant (Rockmill and Roeder 1990, 1991). The mek1-as mutant allows inhibition of Mek1 kinase activity after DSBs have formed. In a dmc1deficient strain background, inhibition of Mek1 kinase activity (by addition of inhibitor to mek1-as cells) allowed meiotic DSBs to be efficiently repaired by recombination with the sister chromatid (Wan et al. 2004). This result suggests that interhomolog partner choice depends on a block to intersister recombination. This work extended previous observations showing that DSBs do not accumulate in a dmc1 mek1 double mutant (Xu et al. 1997) and is consistent with the previously mentioned data on the effects of red1 mutations. Other data suggest that Mek1 must be recruited to the axes by Red1 and Hop1 in order for its kinase activity to promote interhomolog partner choice (Wan et al. 2004). Phosphorylation of a Mek1 substrate in the neighborhood of the recombination event is likely required to promote interhomolog partner choice by blocking intersister recombination. The role of an intersister block to strand invasion in normal meiosis is also suggested by isolation of recessive red 1 and mek 1 alleles in a screen for mutations that elevate unequal intersister recombination (Thompson and Stahl 1999).

Certain data raise the possibility that the axis-associated proteins and Hedl function in the same pathway. Hedl was isolated as a high-dosage suppressor of a coldsensitive allele of Red1 (Tsubouchi and Roeder 2006). High-dosage suppression by Hed1 is specific for the coldsensitive allele, consistent with a direct interaction. Furthermore, the fact that DSBs are efficiently repaired in mek1-as dmc1 mutants suggests that the ability of Hed1 to inhibit Rad51 depends on Mek1 kinase activity (Wan et al. 2004). One intriguing possibility is that the activity of Hed1 could be controlled by Mek1 phosphorylation. However, even if this proves to be the case, Hed1 is unlikely to be the only activity controlled by Mek1, because less interhomolog recombination occurs in $d m c 1$ 
red1 and dmc1 mek1 (Schwacha and Kleckner 1997. Bishop et al. 1999; Niu et al. 2005) than in dmc1 hed1 (Tsubouchi and Roeder 2006). Further characterization of the interplay between the Red1 pathway and Hed1 should be illuminating.

\section{A model for the contribution of Dmc1 and Rad51 to interhomolog partner choice}

EM images of axial elements and recombination nodules in plants led Zickler and Kleckner (1999) to propose that the recombinase complex is a rigid structure that projects away from the axial element during the recombinase search for target sequences on the homologous chromosome. We imagine that such architecture could be responsible for suppression of intersister recombination (Fig. 1). The structural restraint imposed on the complex could function in a manner analogous to the cone collars used by veterinarians to prevent dogs from biting themselves. Such collars do not prevent the wearer from biting other dogs. The structural integrity of a collar-like scaffold may depend upon phosphorylation
A

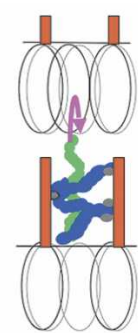

D

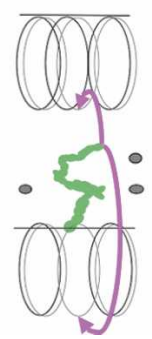

B

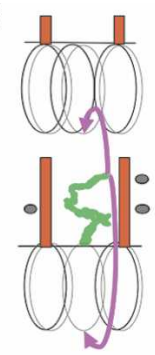

E

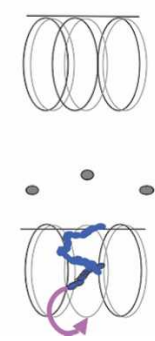

C

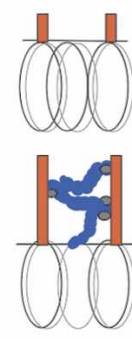

$\mathrm{F}$

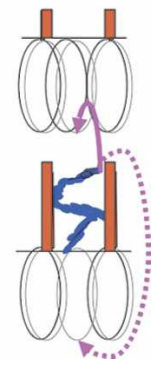

Figure 1. Interhomolog vs. intersister bias during meiotic recombination. (Black/gray loops) Sister chromatids; (red bars) Mek1 phosphorylation-dependent protein scaffold; (green) Dmc1 filament; (blue) Rad51 filament; (black line) axial element; (gray oval) Hed1. (A) Wild type. An active Dmc1 filament is directed away from the sister chromatid by a scaffold composed of axial proteins and inactive Rad51. (B) rad51 mutant. Dmcl is not supported by the scaffold and therefore has reduced interhomolog specificity. $(C) d m c 1$ mutant. Rad51 is inhibited by Hed1. $(D)$ red1 rad51 double mutant. The scaffold is absent. The consequence is the same as in the rad51 mutant. $(E)$ red1 dmc1 double mutant. Hed1 does not function. Rad51 lacks structural support allowing access to the sister chromatid, which is the default target. $(F)$ hed1 dmc1. In the absence of Hed1, Rad51 is activated. Constraint limiting intersister recombination is retained resulting in high levels of interhomolog recombination. of a Mek1 substrate. The observation that Rad51 is needed for interhomolog preference, even though its activity is likely to be suppressed by Hed1, suggests the role of the Rad51 filament could be to provide structural support to Dmc1 (Tsubouchi and Roeder 2006), with the help of the phosphorylated form of the Mek1 substrate. In other words, the Dmcl filament could be bolstered by a structure dependent on interaction between $\operatorname{Rad} 51$ and the Mek1 substrate.

It was previously suggested that Rad51 might load on one end formed by a DSB while Dmcl loads on the other (Shinohara et al. 2000). This hypothetical configuration works well with the present proposal, although other configurations allowing for support of Dmc1 by Rad51 can be imagined as well. The proposed configuration provides an explanation for the effects of several mutations. In a rad51 mutant, the unbuttressed Dmc1 filament would have an increased tendency to target the sister chromatid. In a $d m c 1$ mutant, the architecture pointing Rad51 away from the sister is intact and Hed1 inhibits Rad51's invasion activity and thus very little strand invasion occurs. A dmc1 hed1 double mutant would also have an intact constraining architecture, but Rad51 would be capable of promoting invasion as its strand exchange activity is now uninhibited (the results suggest that inactivation of Hed1 does not fully suppress Dmc1 deficiency and thus some aberrant intersister partner choice may occur in the double mutant). In a dmc1 mutant lacking the Mek1 substrate (or any of the axial proteins required for normal function of the Mek1 kinase), the Rad51 filament would no longer be confined. This filament would therefore target the sister chromatid preferentially as in mitotic recombination.

The model described above does not attempt to incorporate a number of aspects of meiotic regulation that are likely to be influenced by interplay of recombinases. For example, recent observations suggest that the decision of whether or not a strand invasion event will yield a reciprocal crossover recombinant or a noncrossover recombinant is made prior to or during strand invasion (for review, see Bishop and Zickler 2004). In addition, the restriction of strand invasion to an allelic as opposed to an ectopic target sequence is also influenced by recombinases, and this restriction may be related to that limiting intersister recombination (Grushcow et al. 1999; Thompson and Stahl 1999; Henry et al. 2006).

\section{Two phases of meiotic DSB repair?}

A possible scenario that could explain an advantage to loading an inhibited form of Rad51 on DSBs is suggested by data from experiments in which cells in meiotic prophase are returned to mitotic growth (Bishop et al. 1992, 1999; Zenvirth et al. 1997). In the return to growth protocol, a dmc1 mutant retains full viability, and DSBs that are unrepaired as long as cells are kept on meiotic medium are rapidly repaired when cells are returned to growth (Schwacha and Kleckner 1997; Zenvirth et al. 1997). This repair is associated with disappearance of axial and synaptic structures. In contrast, rad51 mutants 
lose viability in the return to growth protocol, even though they display a relatively modest defect in meiotic DSB repair compared with a $d m c 1$ mutant, (Shinohara et al. 1992, 1997). These findings raise the interesting possibility that the strand exchange activity of Rad51 is important during normal meiosis, but that this activity is used to "clean up" a small fraction of DSBs left unrepaired by the interhomolog recombinase machinery. For example, Hed1 may initially prevent Rad51-dependent strand invasion during the initial "reaching out" phase (during which intersister recombination is suppressed). The cost of restricting partner choice may be a reduction in the absolute efficiency of break repair. Such a problem could be overcome by eventually releasing restrictions, thereby allowing residual DSBs to be repaired in late prophase. Release of Rad51 activity, mediated by removal of Hed1, could allow repair of breaks at sites where Dmc1 fails to load. Alternatively, or in addition, disassembly of axial constraints at the end of prophase could activate clean-up of residual DSBs via intersister recombination.

\section{Evolutionary perspective}

Molecular evolutionary studies have proven that the duplication that formed Rad51 and Dmc1 was an ancient event, one that occurred early in eukaryotic evolution (Stassen et al. 1997; Ramesh et al. 2005). Mutant analysis indicates that Dmcl plays an important role in meiosis in most organisms that possess it. Nonetheless, genome sequencing projects led to the finding that Dmcl (and its accessory factors Hop2 and Mnd1) has been independently lost by more than one lineage, including Diptera and nematodes (Ramesh et al. 2005). How can this be explained? We propose that the acquisition of interhomolog activity by Dmc1 drove divergence of the two proteins. For example, Dmcl may have evolved to form stiffer filaments than Rad51 such that its ability to project broken ends away from sister pairs was enhanced. Suppression of Rad51 strand exchange activity by Hed1 may have facilitated evolution of Dmcl's interhomolog activity, at least in budding yeast. Once the interhomolog activity of Dmcl was established, improvement/reinforcement of the projection architecture may have occurred via selection of changes in neighboring proteins, including axial components and Rad51. Continuing elaboration of this supporting architecture may have led to a situation in which the presence of Dmcl became dispensable for interhomolog activity in some species. This type of explanation allows for variation in the extent to which interhomolog recombination depends on Dmcl in different species. At one extreme, already mentioned, are organisms that have lost Dmc1 completely. Arabidopsis thaliana may represent the opposite extreme (Couteau et al. 1999). In this plant, Dmc1 appears to be essential for interhomolog crossover formation; in a dmc1 mutant, there are no chiasmata at diplotene; chromosomes condense as intact univalents. This is in contrast to a rad51 mutant, in which it appears that many breaks are left unrepaired at diplotene (Li et al. 2004). Thus, plants may continue to rely more heavily on an ancestral interhomolog activity of Dmc1 than other species. Budding yeast could represent a functionally intermediate situation in which reinforcement of Dmc1's interhomolog activity by the Rad51-axial interactions has resulted in Rad51's acquisition of the ability to substitute fairly well for the interhomolog strand invasion activity of Dmcl.

\section{Acknowledgments}

We thank Nancy Hollingsworth, Michael Lichten, and John Logsdon for helpful comments on this manuscript. We also acknowledge Gareth Jones for stimulating us to think about differences in the role of Dmc1 in different organisms.

\section{References}

Allers, T. and Lichten, M. 2001. Differential timing and control of noncrossover and crossover recombination during meiosis. Cell 106: $47-57$.

Baumann, P., Benson, F.E., and West, S.C. 1996. Human Rad51 protein promotes ATP-dependent homologous pairing and strand transfer reactions in vitro. Cell 87: 757-766.

Benson, F.E., Stasiak, A., and West, S.C. 1994. Purification and characterization of the human Rad51 protein, an analogue of E. coli RecA. EMBO I. 13: 5764-5771.

Bishop, D.K. 1994. RecA homologs Dmc1 and Rad51 interact to form multiple nuclear complexes prior to meiotic chromosome synapsis. Cell 79: 1081-1092.

Bishop, D.K. and Zickler, D. 2004. Early decision: Meiotic crossover interference prior to stable strand exchange and synapsis. Cell 117: 9-15.

Bishop, D.K., Park, D., Xu, L., and Kleckner, N. 1992. DMC1: A meiosis-specific yeast homolog of $E$. coli recA required for recombination, synaptonemal complex formation, and cell cycle progression. Cell 69: 439-456.

Bishop, D.K., Nikolski, Y., Oshiro, J., Chon, J., Shinohara, M., and Chen, X. 1999. High copy number suppression of the meiotic arrest caused by a $d m c 1$ mutation: REC114 imposes an early recombination block and RAD54 promotes a DMC1-independent DSB repair pathway. Genes Cells 4: 425-444.

Borner, G.V., Kleckner, N., and Hunter, N. 2004. Crossover/ noncrossover differentiation, synaptonemal complex formation, and regulatory surveillance at the leptotene/zygotene transition of meiosis. Cell 117: 29-45.

Bugreev, D.V., Golub, E.I., Stasiak, A.Z., Stasiak, A., and Mazin, A.V. 2005. Activation of human meiosis-specific recombinase Dmc1 by Ca2+. J. Biol. Chem. 280: 26886-26895.

Chen, Y.K., Leng, C.H., Olivares, H., Lee, M.H., Chang, Y.C., Kung, W.M., Ti, S.C., Lo, Y.H., Wang, A.H., Chang, C.S., et al. 2004. Heterodimeric complexes of Hop2 and Mnd1 function with Dmcl to promote meiotic homolog juxtaposition and strand assimilation. Proc. Natl. Acad. Sci. 101: 1057210577.

Couteau, F., Belzile, F., Horlow, C., Grandjean, O., Vezon, D., and Doutriaux, M.P. 1999. Random chromosome segregation without meiotic arrest in both male and female meiocytes of a dmc1 mutant of Arabidopsis. Plant Cell 11: 16231634.

Gasior, S.L., Olivares, H., Ear, U., Hari, D.M., Weichselbaum, R., and Bishop, D.K. 2001. Assembly of RecA-like recombinases: Distinct roles for mediator proteins in mitosis and meiosis. Proc. Nat1. Acad. Sci. 98: 8411-8418. 
Grushcow, J.M., Holzen, T.M., Park, K.J., Weinert, T., Lichten, M., and Bishop, D.K. 1999. Saccharomyces cerevisiae checkpoint genes MEC1, RAD17 and RAD24 are required for normal meiotic recombination partner choice. Genetics 153: $607-620$

Hayase, A., Takagi, M., Miyazaki, T., Oshiumi, H., Shinohara, M., and Shinohara, A. 2004. A protein complex containing Mei5 and Sae 3 promotes the assembly of the meiosis-specific RecA homolog Dmc1. Cell 119: 927-940.

Henry, J.M., Camahort, R., Rice, D.A., Florens, L., Swanson, S.K., Washburn, M.P., and Gerton, J.L. 2006. Mnd1/Hop2 facilitates Dmc1-dependent interhomolog crossover formation in meiosis of budding yeast. Mol. Cell. Biol. 26: 29132923.

Hong, E.L., Shinohara, A., and Bishop, D.K. 2001. Saccharomyces cerevisiae Dmc1 protein promotes renaturation of single-strand DNA (ssDNA) and assimilation of ssDNA into homologous super-coiled duplex DNA. J. Biol. Chem. 276: 41906-41912.

Hunter, N. and Kleckner, N. 2001. The single-end invasion: An asymmetric intermediate at the double-strand break to double-holliday junction transition of meiotic recombination. Cell 106: 59-70.

Jackson, J.A. and Fink, G.R. 1985. Meiotic recombination between duplicated genetic elements in Saccharomyces cerevisiae. Genetics 109: 303-332.

Kadyk, L.C. and Hartwell, L.H. 1992. Sister chromatids are preferred over homologs as substrates for recombinational repair in Saccharomyces cerevisiae. Genetics 132: 387-402.

Kinebuchi, T., Kagawa, W., Enomoto, R., Tanaka, K., Miyagawa, K., Shibata, T., Kurumizaka, H., and Yokoyama, S. 2004. Structural basis for octameric ring formation and DNA interaction of the human homologous-pairing protein Dmc1. Mol. Cell 14: 363-374.

Lee, M.H., Chang, Y.C., Hong, E.L., Grubb, J., Chang, C.S., Bishop, D.K., and Wang, T.F. 2005. Calcium ion promotes yeast Dmcl activity via formation of long and fine helical filaments with single-stranded DNA. J. Biol. Chem. 280: 40980-40984.

Li, Z., Golub, E.I., Gupta, R., and Radding, C.M. 1997. Recombination activities of HsDmcl protein, the meiotic human homolog of RecA protein. Proc. Natl. Acad. Sci. 94: 1122111226.

Li, W., Chen, C., Markmann-Mulisch, U., Timofejeva, L., Schmelzer, E., Ma, H., and Reiss, B. 2004. The Arabidopsis AtRAD51 gene is dispensable for vegetative development but required for meiosis. Proc. Natl. Acad. Sci. 101: 1059610601.

Mao-Draayer, Y., Galbraith, A.M., Pittman, D.L., Cool, M., and Malone, R.E. 1996. Analysis of meiotic recombination pathways in the yeast Saccharomyces cerevisiae. Genetics 144: 71-86.

Masson, J.Y., Davies, A.A., Hajibagheri, N., Van Dyck, E., Benson, F.E., Stasiak, A.Z., Stasiak, A., and West, S.C. 1999. The meiosis-specific recombinase $\mathrm{hDmcl}$ forms ring structures and interacts with hRad51. EMBO J. 18: 6552-6560.

Niu, H., Wan, L., Baumgartner, B., Schaefer, D., Loidl, J., and Hollingsworth, N.M. 2005. Partner choice during meiosis is regulated by Hop1-promoted dimerization of Mek1. Mol. Biol. Cell 16: 5804-5818.

Ogawa, T., Yu, X., Shinohara, A., and Egelman, E.H. 1993. Similarity of the yeast Rad51 filament to the bacterial RecA filament. Science 259: 1896-1899.

Passy, S.I., Yu, X., Li, Z., Radding, C.M., Masson, J.Y., West, S.C., and Egelman, E.H. 1999. Human Dmc1 protein binds
DNA as an octameric ring. Proc. Natl. Acad. Sci. 96: 1068410688.

Pecina, A., Smith, K.N., Mezard, C., Murakami, H., Ohta, K., and Nicolas, A. 2002. Targeted stimulation of meiotic recombination. Cell 111: 173-184.

Ramesh, M.A., Malik, S.B., and Logsdon Jr., J.M. 2005. A phylogenomic inventory of meiotic genes; evidence for sex in Giardia and an early eukaryotic origin of meiosis. Curr. Biol. 15: 185-191.

Rockmill, B. and Roeder, G.S. 1990. Meiosis in asynaptic yeast. Genetics 126: 563-574.

- 1991. A meiosis-specific protein kinase homolog required for chromosome synapsis and recombination. Genes \& Dev. 5: 2392-2404.

Rockmill, B., Sym, M., Scherthan, H., and Roeder, G.S. 1995. Roles for two RecA homologs in promoting meiotic chromosome synapsis. Genes \& Dev. 9: 2684-2695.

Schwacha, A. and Kleckner, N. 1994. Identification of joint molecules that form frequently between homologs but rarely between sister chromatids during yeast meiosis. Cell 76: 5163.

. 1997. Interhomolog bias during meiotic recombination: Meiotic functions promote a highly differentiated interhomolog-only pathway. Cell 90: 1123-1135.

Sehorn, M.G., Sigurdsson, S., Bussen, W., Unger, V.M., and Sung, P. 2004. Human meiotic recombinase Dmc1 promotes ATP-dependent homologous DNA strand exchange. Nature 429: 433-437.

Shinohara, A., Ogawa, H., and Ogawa, T. 1992. Rad51 protein involved in repair and recombination in $S$. cerevisiae is a RecA-like protein. Cell 69: 457-470.

Shinohara, A., Gasior, S., Ogawa, T., Kleckner, N., and Bishop, D.K. 1997. Saccharomyces cerevisiae RECA homologues RAD51 and DMC1 have both distinct and overlapping roles in meiotic recombination. Genes Cells 2: 615-629.

Shinohara, M., Gasior, S.L., Bishop, D.K., and Shinohara, A. 2000. Tid1/Rdh54 promotes colocalization of Rad51 and Dmc1 during meiotic recombination. Proc. Natl. Acad. Sci. 97: 10814-10819.

Shinohara, M., Sakai, K., Shinohara, A., and Bishop, D.K. 2003. Crossover interference in Saccharomyces cerevisiae requires a TID1/RDH54- and DMC1-dependent pathway. Genetics 163: $1273-1286$.

Stassen, N.Y., Logsdon Jr., J.M., Vora, G.J., Offenberg, H.H., Palmer, J.D., and Zolan, M.E. 1997. Isolation and characterization of rad51 orthologs from Coprinus cinereus and Lycopersicon esculentum, and phylogenetic analysis of eukaryotic recA homologs. Curr. Genet. 31: 144-157.

Story, R.M., Bishop, D.K., Kleckner, N., and Steitz, T.A. 1993. Structural relationship of bacterial RecA proteins to recombination proteins from bacteriophage T4 and yeast. Science 259: 1892-1896.

Sung, P. 1994. Catalysis of ATP-dependent homologous DNA pairing and strand exchange by yeast Rad51 protein. Science 265: 1241-1243.

Tan, T.L., Kanaar, R., and Wyman, C. 2003. Rad54, a Jack of all trades in homologous recombination. DNA Repair (Amst.) 2: 787-794.

Tarsounas, M., Morita, T., Pearlman, R.E., and Moens, P.B. 1999. RAD51 and DMC1 form mixed complexes associated with mouse meiotic chromosome cores and synaptonemal complexes. J. Cell Biol. 147: 207-220.

Thompson, D.A. and Stahl, F.W. 1999. Genetic control of recombination partner preference in yeast meiosis.: Isolation and characterization of mutants elevated for meiotic un- 
equal sister-chromatid recombination. Genetics 153: 621641.

Tsubouchi, H. and Roeder, G.S. 2003. The importance of genetic recombination for fidelity of chromosome pairing in meiosis. Dev. Cell 5: 915-925.

. 2004. The budding yeast Mei5 and Sae3 proteins act together with Dmcl during meiotic recombination. Genetics 168: 1219-1230.

. 2006. Budding yeast Hed1 down-regulates the mitotic recombination machinery when meiotic recombination is impaired. Genes \& Dev. (this issue).

Wan, L., de los Santos, T., Zhang, C., Shokat, K., and Hollingsworth, N.M. 2004. Mek1 kinase activity functions downstream of RED1 in the regulation of meiotic double strand break repair in budding yeast. Mol. Biol. Cell 15: 11-23.

Xu, L., Weiner, B.M., and Kleckner, N. 1997. Meiotic cells monitor the status of the interhomolog recombination complex. Genes \& Dev. 11: 106-118.

Zenvirth, D., Loidl, J., Klein, S., Arbel, A., Shemesh, R., and Simchen, G. 1997. Switching yeast from meiosis to mitosis: Double-strand break repair, recombination and synaptonemal complex. Genes Cells 2: 487-498.

Zickler, D. and Kleckner, N. 1999. Meiotic chromosomes: Integrating structure and function. Annu. Rev. Genet. 33: 603754. 


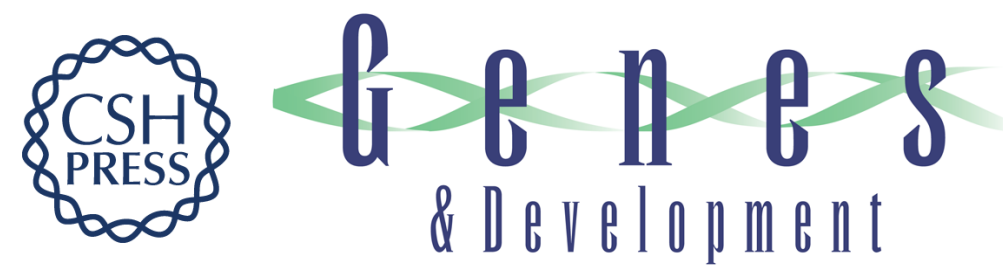

\section{Red-Hed regulation: recombinase Rad51, though capable of playing the leading role, may be relegated to supporting Dmc1 in budding yeast meiosis}

Sean Sheridan and Douglas K. Bishop

Genes Dev. 2006, 20:

Access the most recent version at doi:10.1101/gad.1447606

\section{References This article cites 52 articles, 29 of which can be accessed free at: http://genesdev.cshlp.org/content/20/13/1685.full.html\#ref-list-1}

\section{License}

Email Alerting
Service

Receive free email alerts when new articles cite this article - sign up in the box at the top right corner of the article or click here.

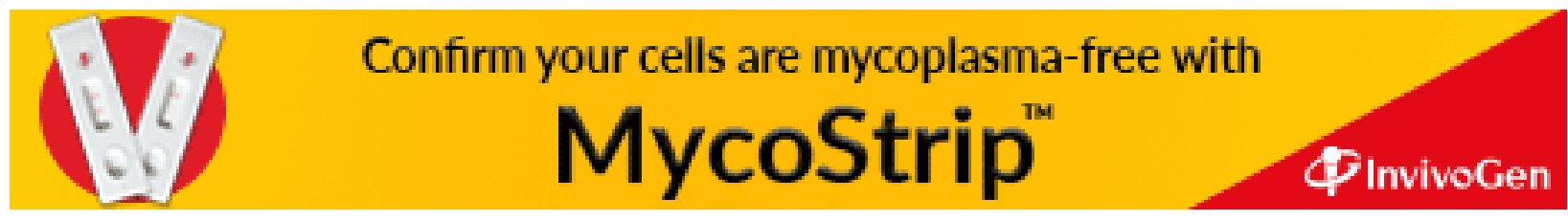

